\title{
Acquisition and retention of negative patterning in hippocampal-lesioned rats
}

\author{
DARLENE M. SKINNER and DEREK VAN DER KOOY \\ University of Toronto, Toronto, Ontario, Canada
}

\begin{abstract}
In this series of experiments, we examined whether animals with aspiration lesions of the hippocampus could retain a negative patterning task learned preoperatively (Experiment 1) or acquire a negative patterning task postoperatively (Experiment 2). In a negative patterning task, each of two single cues is followed by an unconditioned stimulus $(\mathrm{A}+, \mathrm{B}+)$, while the two cues presented together are not followed by the unconditioned stimulus ( $\mathrm{AB}-$ ). Rats were exposed to a novel context and allowed to drink a saccharin solution for 15 min on $\mathrm{AB}$ - trials. On A+trials, the rats were exposed to saccharin alone, and on $B+$ trials, the rats were exposed to the novel context with a water bottle and were given an injection of $\mathrm{LiCl}$ in both cases. The rats showed aversions to the context and saccharin alone but consumed large amounts of the saccharin when the two cues were presented together. While hippocampal-lesioned rats showed some deficits on retention tests, they showed good acquisition of the negative patterning task.
\end{abstract}

Converging evidence from human, primate, and rodent studies points to a role for the hippocampal formation in learning and memory (Cohen \& Squire, 1980; McDonald \& White, 1993; Morris, Garrud, Rawlins, \& O'Keefe, 1982; Ridley, Timothy, Maclean, \& Baker, 1995; Squire, 1986). Yet although most would agree that the hippocampus is involved in the acquisition and/or storage of information, there is considerable debate over the exact role that the hippocampus plays (Eichenbaum, Otto, \& Cohen, 1992; Hirsh, 1974; O’Keefe \& Nadel, 1979; Olton, Becker, \& Handelmann, 1980; Squire, 1986; Sutherland \& Rudy, 1989). There are a number of theories of hippocampal function. One long standing theory suggests that the hippocampus is preferentially involved in spatial learning (O'Keefe \& Nadel, 1979). Indeed, considerable support for this theory has accumulated over the years from many animal studies (Eichenbaum, Stewart, \& Morris, 1990; Morris et al., 1982; Morris, Schenk, Tweedie, \& Jarrard, 1990; Sutherland \& Rudy, 1988; Sutherland, Whishaw, $\&$ Kolb, 1983). Yet despite the wide attention and support that this theory has received, there are now many examples of nonspatial tasks that are disrupted by hippocampal lesions (Aggleton, Kentridge, \& Sembi, 1991; Eichenbaum, Fagan, Mathews, \& Cohen, 1988; Raffaele \& Olton, 1988; Sutherland \& McDonald, 1990; Wan, Pang, \& Olton, 1994; Yee \& Rawlins, 1994). This has led to the speculation that the hippocampus is involved in the general processing of all types of information and that spa-

D. van der Kooy is in the Department of Anatomy and Cell Biology at Toronto. Correspondence should be addressed to D. M. Skinner, who is now at the Department of Psychology: Experimental, 229 Sociology/Psychology Building, Duke University, Durham, NC 277080086 (e-mail: dskinner@psych.duke.edu).

-Accepted by previous editor, Paul E. Gold tial learning is just one example of configural, relational, or declarative learning in which an animal has to learn the relationship among multiple cues (Eichenbaum et al., 1992, 1994; Sutherland \& Rudy, 1989).

In recent years, the configural association theory (Sutherland \& Rudy, 1989) has received a great deal of attention. This theory explicitly states that the hippocampus is involved when an animal has to learn the relationship among multiple cues, regardless of their nature, and that simple associations involving only two elements, as in Pavlovian conditioning, can be solved in the absence of an intact hippocampus. Because this theory is explicit and is easy to test, and because it accounts for spatial deficits as well as a great deal of other data, it has provided the rationale for many experiments (Alvarado \& Rudy, 1995; Davidson, McKernan, \& Jarrard, 1993; Gallagher \& Holland, 1992; Rudy \& Sutherland, 1989; Sutherland \& McDonald, 1990; Whishaw \& Tomie, 1991, 1995; Whishaw, Tomie, \& Kolb, 1992). These tests of configural theory have yielded conflicting results, and there are now several examples of configural tasks that can be acquired in the absence of an intact hippocampus (Davidson et al., 1993; Gallagher \& Holland, 1992; Whishaw \& Tomie, 1991). While it is always possible that without a hippocampus an animal may adopt a nonconfigural strategy to solve an apparently configural task, it is up to the researcher to design a task that at least a priori has no obvious simple association solution. Such tasks include transverse patterning, biconditional discriminations, and negative patterning; all of these problems have nonlinear solutions (Alvarado \& Rudy, 1992; Rudy \& Sutherland, 1989; Sutherland \& Rudy, 1989; Woodbury, 1943). In a task with a linear solution, the response reflects the sum of excitatory and inhibitory associations between the component elements and the reinforcer. $A$ task such as the negative patterning problem $(\mathrm{A}+, \mathrm{B}+, \mathrm{AB}-)$ requires a nonlinear solution, 
because the sum of excitatory strength of each of the elements when presented in compound is actually lower than that of the individual cues. A linear strategy would involve responding more on $A B$ trials, as a result of simple summation, and this clearly is not the correct solution to the negative patterning problem.

For the present series of experiments, we designed a negative patterning task using cues that we had previously used in a conditional discrimination (Skinner, Martin, Harley, et al., 1994; Skinner, Martin, Pridgar, \& van der Kooy, 1994). In the conditional task, one distinct context predicted a flavor-toxin pairing, whereas a second, distinct context predicted the same flavor in the absence of the toxin. Rats with large aspiration lesions of the hippocampus could acquire the task but at a slower rate than did sham controls. We argued (Skinner, Martin, Harley, et al., 1994) that the impairment was due to the nature of the contextual cues used (cf. Good \& Honey, 1991; Honey \& Good, 1993; Kim \& Fanselow, 1992; Selden, Everitt, Jarrard, \& Robbins, 1991; Young, Bohenek, \& Fanselow, $1994)$ and not to deficits in learning the conditional strategy. Behavioral tests revealed that both lesioned and sham animals were solving the task in the same manner, one that did not depend solely on simple associations. These data led us to propose that conditional discrimination learning was a hippocampal-independent configural task. However, unlike transverse patterning and negative patterning tasks, conditional discriminations can be solved with the use of a linear strategy (Alvarado \& Rudy, 1992). For example, a rat may form a simple association between a context and $\mathrm{LiCl}$ and sum this with a flavor-toxin association. In the present study, we designed a task that Sutherland \& Rudy (1989) had defined as configural, using the same contextual, flavor, and toxin cues. In Experiment 1 , lesioned rats were tested for retention of a preoperatively learned negative patterning task. In Experiment 2, hippocampal-lesioned rats were trained postoperatively to acquire a negative patterning task.

\section{EXPERIMENT 1}

The purpose of the present experiment was to design a negative patterning task with the same cues that we had previously employed in a conditional discrimination paradigm (Skinner, Martin, Harley, et al., 1994; Skinner, Martin, Pridgar, \& van der Kooy, 1994). In a negative patterning paradigm, each of two single cues is followed by an unconditioned stimulus (US) $(\mathrm{A}+, \mathrm{B}+)$, whereas the two cues presented together are not followed by the US $(\mathrm{AB}-)$. In the present design, animals were exposed to a saccharin solution in a distinct environment, in a manner similar to the way in which we train conditional discriminations. Following removal of the saccharin, the animals were returned to their home cages ( $\mathrm{AB}$ - trials). Exposure to the saccharin alone (in the home cage) or to the distinct environment with a water bottle were both followed by an injection of $\mathrm{LiCl}(\mathrm{A}+$ and $\mathrm{B}+$ trials, respectively). Thus, the context was identical on $\mathrm{AB}-$ and $\mathrm{B}+$ trials except for the actual taste of the saccharin solution. Since tap water was given in the home cage each afternoon, all single elements of the task were ambiguous. Rats were trained on this task and were tested for retention after aspiration lesions of the hippocampus.

\section{Method}

Subjects. Ten male Wistar rats (Charles River Breeders, Quebec, Canada), weighing approximately $300 \mathrm{~g}$ at the start of the experiment were singly housed in hanging wire cages and maintained on a 12:12-h light:dark cycle. A water-deprivation schedule consisting of $60-\mathrm{min}$ access to tap water each afternoon was implemented 1 week after the animals' arrival in the lab and 1 week prior to behavioral training. The rats were allowed free access to standard lab chow.

Negative patterning acquisition. All subjects were trained on a negative patterning task with one trial per day. On AB-trials, the rats were placed in the distinct box 15 min prior to $15-\mathrm{min}$ access to a novel $0.1 \%$ saccharin solution. After removal of the saccharin, the rats were returned to the home cage. On A+ trials, the rats were given $15 \mathrm{~min}$ of exposure to the same saccharin solution while in the home cage. Removal of the saccharin was followed by an intraperitoneal injection of $100-\mathrm{mg} / \mathrm{kg} \mathrm{LiCl}$ dissolved in $3-\mathrm{ml} /$ $\mathrm{kg}$ physiological saline. On $\mathrm{B}+$ trials, the rats were exposed to the distinct box $15 \mathrm{~min}$ prior to $15-\mathrm{min}$ access to tap water. The rats were then injected with $\mathrm{LiCl}$ and returned to the home cage. For half the rats, the training environment was a black box with a Plexiglas floor and a slight smell of vinegar. For the remainder of the rats, the training environment was a white box with wire mesh floors covered with wood chips. The animals were trained on the negative patterning task for 49 cycles of 3 trials $(\mathrm{AB}-, \mathrm{A}+, \mathrm{B}+)$ for a total of 147 trials with only 1 trial given per day. The training phase was organized into blocks of 10 cycles, with the last block having 9 cycles.

Surgery. Upon completion of acquisition training, 5 rats underwent aspiration lesions of the hippocampus as outlined in detail in Skinner, Martin, Harley, et al. (1994). Briefly, the rats were anesthetized with sodium pentobarbital $(65 \mathrm{mg} / \mathrm{kg}$ i.p.) and placed in a stereotaxic instrument. The skull overlying the area to be aspirated was removed with a surgical drill. The hippocampus was removed bilaterally by aspiration, using a glass pipette attached to a vacuum aspirator. The remaining 5 animals underwent the same procedure, but only the cortex overlying the hippocampus was removed. Since all the rats had acquired the task preoperatively, random assignment to either the lesion or the sham group resulted in preoperative behavior that was similar in the lesion and sham groups. The rats were allowed to recover for 1 week before retention testing.

Histology. Upon completion of behavioral testing, all the rats were deeply anesthetized with sodium pentobarbital and perfused through the heart with saline followed by $10 \%$ formalin. The brains were removed and stored in $15 \%$ sucrose, and cryostat sections were cut. The sections were mounted on slides and stained with cresyl violet to verify the placement and extent of the lesions.

Retention. One week after surgery, the animals were tested for retention of the negative patterning task learned preoperatively. The retention testing was identical to the initial acquisition training. The animals were retrained for three blocks of 10 trials.

\section{Results}

Initial acquisition. Acquisition was apparent by the second block of 10 cycles (Figure 1). A $10 \times 3$ (cycles $\times$ trials) analysis of variance (ANOVA) over the first block of 10 cycles revealed a significant main effect of cycles $[F(9,90)=11.61, p<.05]$ and a significant cycles $\times$ trials 


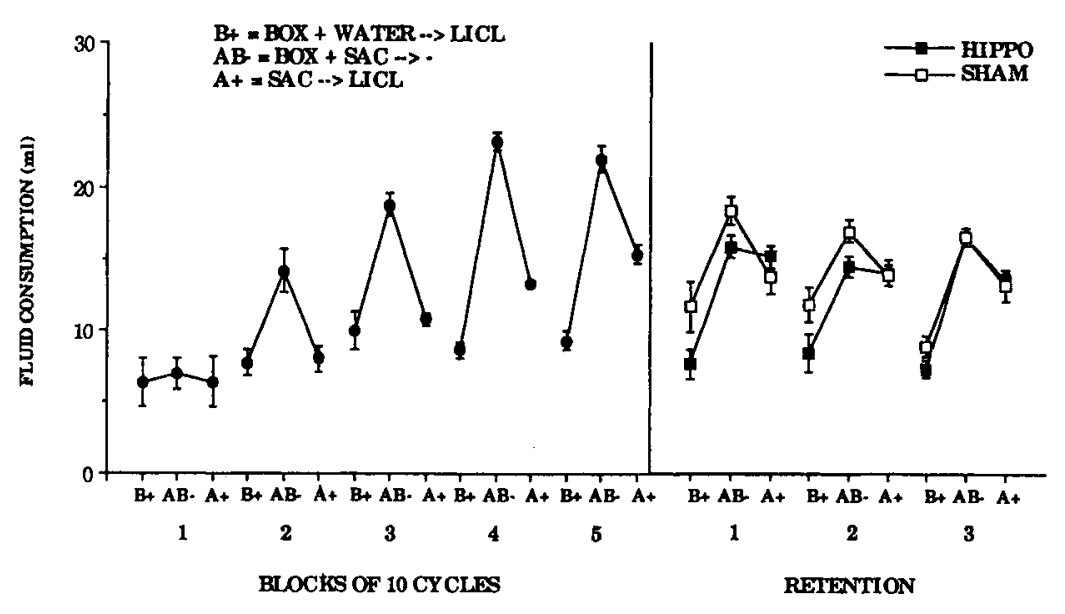

Figure 1. Left panel shows acquisition of negative patterning in unlesioned rats. Training was divided into blocks of 10 cycles. $\mathrm{A}+=\mathrm{Sac} \rightarrow \mathrm{LiCl} ; \mathrm{B}+=\mathrm{Box}+$ Water $\rightarrow \mathrm{LiCl} ; \mathrm{AB}-=\mathrm{Box}+\mathrm{Sac} \rightarrow-(n=10$ rats $)$. Right panel shows postoperative retention of the same task. Retraining was divided into three blocks of 10 cycles $(n=4$ lesioned and $n=3$ sham animals).

interaction $[F(18,180)=4.43, p<.05]$. The significant interaction reflects the emerging acquisition by some rats in the later cycles of the block. A $10 \times 3$ ANOVA over the second block of 10 cycles revealed a main effect of trial type $[F(2,180)=23.20, p<.05]$ and a cycles $X$ trials interaction $[F(18,180)=1.66, p=.05]$ that approached significance, reflecting acquisition of the task. Although comparison of the first two blocks of training in Figure 1 suggests that consumption was not suppressed on $\mathrm{A}+$ and $\mathrm{B}+$ trials but rather increased on $\mathrm{AB}-$ trials, this was not really the case. Consumption on the first block of trials was already at a suppressed level. At the beginning of training, the rats consumed large amounts of fluid, but after the first few $\mathrm{LiCl}$ injections, the rats showed general suppression across all trials. Only with further training did the rats increase consumption on the $\mathrm{AB}$ - trials. By the last block of cycles, an ANOVA revealed only a main effect of trials $[F(2,162)=86.50, p<$ $.05]$. Newman-Keuls tests revealed that consumption on $\mathrm{AB}-$ trials was higher than on $\mathrm{A}+$ and $\mathrm{B}+$ trials $(p s<$ .05 ). In addition, consumption of saccharin on $A+$ trials was higher than consumption of water on $B+$ trials during the last block of 10 cycles. It is unlikely that this effect was due to an unconditioned preference for saccharin over tap water, since it was not seen early in training (first block of Figure 1).

Histology. Two sham lesioned animals and 1 hippocampal lesioned animal died during surgery or early in postoperative training and are excluded from all analyses. All lesioned animals received bilateral damage to the hippocampus proper and subiculum as well as overlying cortex and corpus callosum. In half the lesioned rats, there were variable amounts of sparing in the medial part of the dorsal hippocampus. All lesioned rats had some sparing of ventral hippocampus and subiculum. The sham lesioned rats had damage only to the overlying cortex, with minimal damage to the hippocampus. Figure 2 shows a typical hippocampal aspiration lesion from this experiment.

Postoperative retention. Aspiration lesions of the hippocampus did affect retention of the preoperatively learned negative patterning task but did not completely block performance on the task. A $2 \times 10 \times 3$ (groups $\times$ cycles $x$ trials) ANOVA over the first block of 10 cycles revealed a significant cycles $\times$ trials interaction $[F(18,100)=$ $1.84, p<.05$ ], which reflects some reacquisition of the task. There was also a significant groups $\times$ trials interaction $[F(2,100)=6.16, p<.05]$ but the three-way interaction was not significant $(F<1)$. Newman-Keuls tests on the groups $\times$ trials interaction revealed that the sham group consumed more on $\mathrm{AB}$ - trials than on either the $\mathrm{A}+$ or the $\mathrm{B}+$ trials $(p s<.05)$, which did not differ $(p>$ .05 ). The hippocampal lesioned animals consumed equal amounts of saccharin on $\mathrm{AB}-$ and $\mathrm{A}+$ trials $(p>.05)$, suggesting loss of discriminative performance. However, the rats in this group did consume less water on $\mathrm{B}+$ trials than saccharin on $\mathrm{AB}-$ trials $(p<.05)$, suggesting at least some retention of the preoperatively learned task. An ANOVA over the last block of postoperative retention revealed only a main effect of trial type $[F(2,94)=$ $56.91, p<.05]$. Newman-Keuls tests revealed that both groups consumed more saccharin on $\mathrm{AB}$ - trials than on $\mathrm{A}+$ trials $(p<.05)$, but also that tap water consumption on $\mathrm{B}+$ trials was lower than saccharin consumption on $\mathrm{A}+$ trials $(p<.05)$. One sham lesioned animal died in the late phases of training, so data from this animal are missing from the last three cycles.

\section{Discussion}

Unlesioned rats acquired the negative patterning task quite rapidly. By the end of the second block of training, rats were suppressing fluid consumption on $\mathrm{A}+$ and $\mathrm{B}+$ trials and consuming significantly more on $\mathrm{AB}-$ trials. Although hippocampal lesions disrupted retention of this 

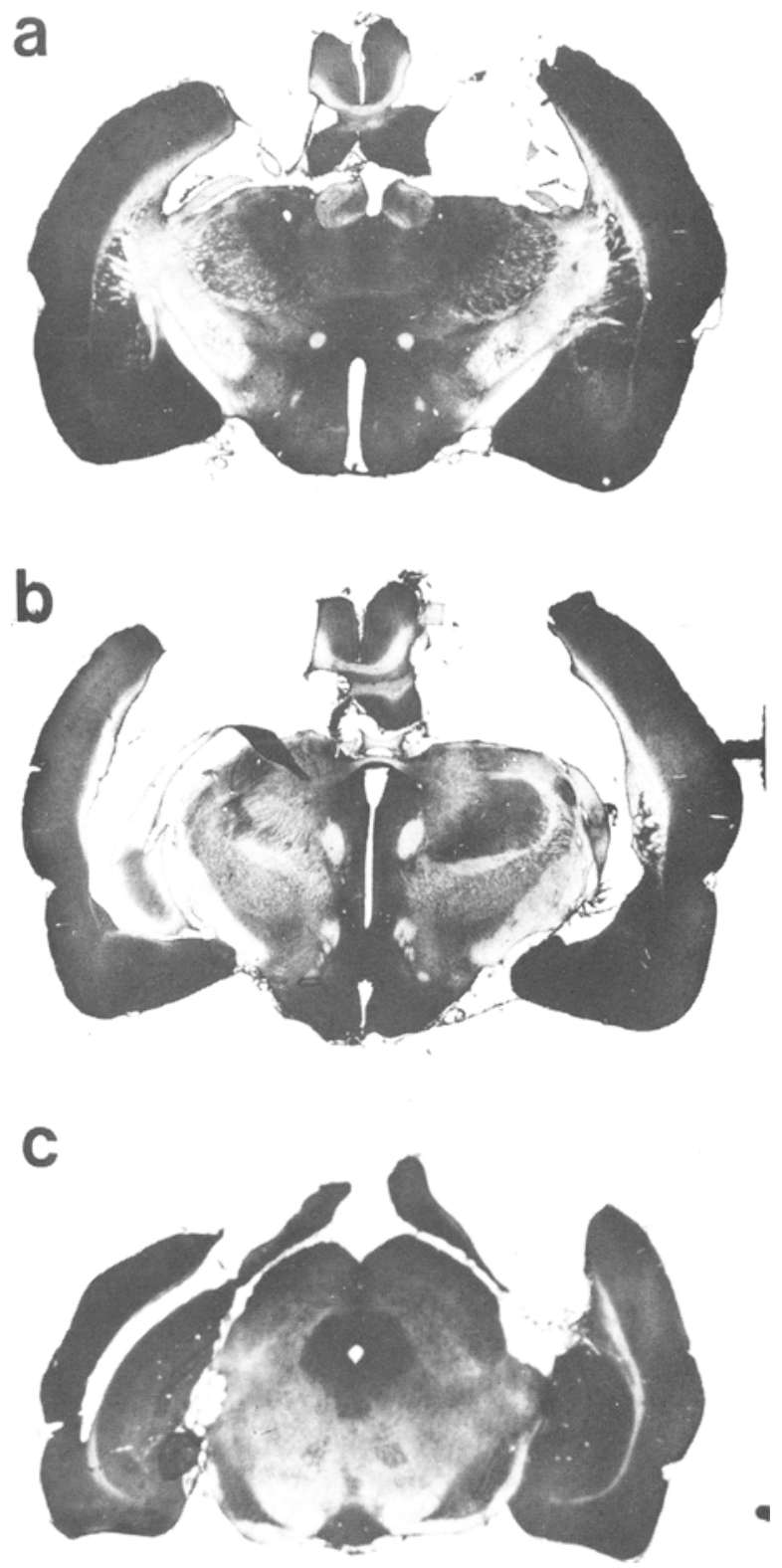

Figure 2. Photomicrographs (rostral to caudal) of coronal sections through the forebrain of a representative rat from the aspiration hippocampal groups in this series of experiments. This specific rat is from Experiment 1.

task, there was clear evidence of some savings. The animals in both groups consumed less fluid on $\mathrm{B}+$ trials than on $\mathrm{AB}$ - trials. In contrast, saccharin consumption was the same in hippocampal lesioned rats on $\mathrm{AB}-$ and $\mathrm{A}+$ trials. This loss of discriminative performance on $A B$ - versus $\mathrm{A}+$ trials in the hippocampal lesioned rats may have been due to interference between these two trial types. However, there was no reason to suspect more interference between these two trials than between $\mathrm{AB}-$ and $\mathrm{B}+$ trials. Indeed, one might have expected more interference between the latter two, given that they had more overlapping features. The contextual cues and the water bottle were identical; only the taste of the fluid was different. With $\mathrm{AB}-$ and
A trials, only the taste of the saccharin solution was the same; all other contextual cues were different.

In spite of the low numbers of subjects in the present experiment, some initial disruption of the preoperatively learned negative patterning task was observed in the hippocampal lesioned rats. We have previously shown that hippocampal lesioned rats were slower than sham controls to acquire a conditional discrimination task using contextual cues very similar to those used in the present negative patterning task (Skinner, Martin, Harley, et al., 1994). In the following experiment, we assessed acquisition of our negative patterning task in hippocampal lesioned animals.

\section{EXPERIMENT 2}

Given some evidence of savings and reacquisition of negative patterning after aspiration lesions of the hippocampus, we assessed acquisition of the negative patterning task to test whether acquisition is more sensitive to hippocampal damage than retention. We have previously found some deficits on context learning with these lesions (Skinner, Martin, Harley, et al., 1994). Hippocampal lesioned animals were slower to learn a context conditional discrimination than sham lesioned controls. They could, however, eventually reach control levels of performance, suggesting that they could use some form of contextual cue(s) to solve the task. Consequently, we trained hippocampal and sham lesioned animals on the conditional component of the task prior to negative patterning training. That is, animals were first given $\mathrm{AB}-$ and $A+$ trials. Once rats had learned to suppress consumption of saccharin in the home cage relative to a novel, distinct environment, we introduced B + trials (tap water in the novel, distinct environment). We decided not to train hippocampal lesioned rats on the negative patterning task from the start, because any impairment we observed might have been due to the nature of the contextual cues used or to a deficit in the negative patterning strategy. By ensuring that lesioned rats could use contextual cues (in the conditional task), we could say that any further impairment was due to a deficit in configural learning.

\section{Method}

Subjects. Sixteen male Wistar rats (Charles River Breeders, Quebec, Canada), weighing approximately $300 \mathrm{~g}$ at the start of the experiment, were singly housed in hanging wire cages and maintained on a 12:12-h light:dark cycle. A water-deprivation schedule consisting of 60-min access to tap water each afternoon was implemented 2 weeks prior to behavioral training. The rats were allowed free access to standard lab chow.

Surgery and histology. All surgical and histological procedures were identical to those of the previous experiment. Eight rats underwent hippocampal aspiration lesions, and 8 rats were given sham lesions. The animals were allowed to recover for 4 weeks before behavioral training was carried out.

Conditional discrimination training. The animals were trained on the conditional component of the negative patterning task prior to negative patterning training. That is, the animals were first given $\mathrm{AB}-$ and $\mathrm{A}+$ trials. We have previously shown that aspiration lesions of the hippocampus impaired acquisition of a condi- 
tional discrimination using contextual cues, but that rats could eventually reach control levels (Skinner, Martin, Harley, et al., 1994). We decided to train rats on the conditional component of the task first to allow them to acquire contextual information before training them on the negative patterning task. On $\mathrm{AB}-$ trials, rats were placed in the distinct box $15 \mathrm{~min}$ prior to 15 -min access to a novel $0.1 \%$ saccharin solution. After removal of the saccharin, the rats were returned to the home cage. On A+trials, the rats were given $15 \mathrm{~min}$ of exposure to the same saccharin solution while they were in the home cage. Removal of the sac.harin was followed by an intraperitoneal injection of $100-\mathrm{mg} / \mathrm{kg} \mathrm{LiCl}$ dissolved in $3-\mathrm{ml} / \mathrm{kg}$ physiological saline. The novel contexts used in this experiment were the same as those used in the previous experiment. The animals were trained on this part of the task for 73 trials with only one trial given per day. There were a total of $17 \mathrm{~A}+$ trials with a varying number of $\mathrm{AB}$ - trials after each $\mathrm{A}+$ trial (for a total of $56 \mathrm{AB}$ - trials). This was done to increase saccharin consumption in some animals that developed a taste aversion to the saccharin solution. For the purpose of statistical analyses, this phase was divided into seventeen 2-day cycles.

Negative patterning training. Upon completion of the discrimination task, the animals were trained on a negative patterning task simply by adding the third trial type to the previous task. That is, in addition to the $A B-$ and $A+$ trials, the animals were now given $\mathrm{B}+$ trials as well. On $\mathrm{B}+$ trials, the rats were exposed to the distinct box $15 \mathrm{~min}$ prior to $15 \mathrm{~min}$ of access to tap water. They were then injected with $\mathrm{LiCl}$ and returned to the home cage. Trials 74-208 constituted this phase of the experiment. For the purpose of statistical analyses, this phase of the experiment was also divided into 17 cycles with 3 trials per cycle. Only the cycles in which the three trial types occurred together were analyzed. More trials of each type were given, but only those that occurred as three successive different trial types were analyzed. One lesioned and one sham lesioned animal died during this phase of the experiment, so there were $n=7$ animals per group.

\section{Results}

Histology. The placements and extents of the lesions were very similar to that seen in the previous experiment (see Figure 2).

Conditional discrimination acquisition. The hippocampal lesioned rats learned the conditional component of the task at the same rate as did the sham controls. Over the course of training, both groups consumed more saccharin on $\mathrm{AB}-$ trials than on $\mathrm{A}+$ trials (see Figure 3, where the first 16 cycles have been divided into blocks of 4 cycles). A $2 \times 17 \times 2$ (groups $\times$ cycles $\times$ trials) ANOVA over the acquisition phase revealed a significant cycles $\times$ trials interaction $[F(16,238)=14.33, p<.05]$, reflecting acquisition of the discrimination, and a significant groups $\times$ trials interaction $[F(1,238)=52.25, p<$ $.05]$. Newman-Keuls tests revealed that the hippocampal lesioned rats consumed less on $\mathrm{AB}$ - trials than did the sham controls $(p<.05)$. Both groups acquired the task at the same rate, as evidenced by an insignificant groups $\times$ cycles $\times$ trials interaction $(F<1)$. By the last cycle of training (Figure 3, right panel), a $2 \times 2$ (groups $\times$ trials) ANOVA revealed only an effect of trials $[F(1,14)=75.39$, $p<.05$ ].

Negative patterning acquisition. One hippocampal and one sham lesioned rat died prior to this phase of the experiment and thus $n=7$ for both groups. Both hippocampal and sham lesioned animals acquired the negative

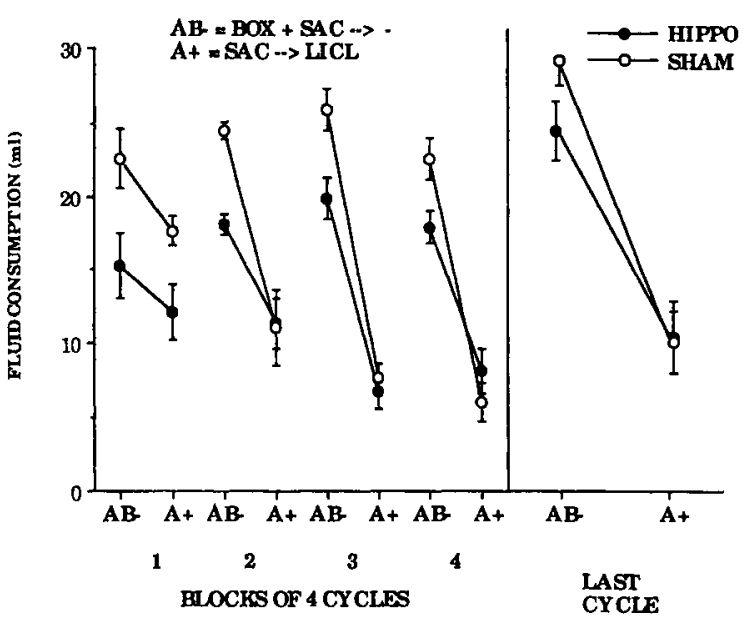

Figure 3. Acquisition of the conditional component of the negative patterning task in hippocampal-lesioned and sham control rats. Left panel divides training into four blocks of four cycles. Right panel shows last cycle of training (Cycle 17). $\mathrm{AB}-=$ $\mathrm{Box}+\mathrm{Sac} \rightarrow-; \mathrm{A}+=\mathrm{Sac} \rightarrow \mathrm{LiCl}(n=8$ lesioned and $n=8$ sham rats).

patterning task. Although the negative patterning task required many more trials than the conditional component, we analyzed only data from cycles in which the three different trial types occurred successively. The order of the trials varied considerably. In some cases, five B+ trials were given in a row. Although tap water consumption decreased over these five trials, analyzing these data did not illuminate the strategy the rats used to solve the task. Conversely, analyzing data from cycles in which the three trial types occurred successively, but not always in the same order, allowed us to ascertain whether or not the rats were solving the negative patterning task. A total of 17 cycles were analyzed (see Figure 4, where the first 16 cycles have been divided into blocks of 4 cycles). A $2 \times$ $17 \times 3$ (groups $\times$ cycles $\times$ trials) ANOVA over the acquisition phase revealed a significant cycles $X$ trials interaction $[F(32,408)=5.04, p<.05]$, reflecting acquisition of the task, and a significant groups $\times$ trials interaction $[F(2,408)=29.01, p<.05]$. Newman-Keuls tests revealed that both groups consumed more fluid on $\mathrm{AB}-$ trials than on either $\mathrm{A}+$ or $\mathrm{B}+$ trials $(p s<.05)$. However, sham lesioned rats consumed more than hippocampal rats on both $\mathrm{AB}-$ and $\mathrm{B}+$ trials $(p s<.05)$. By the last cycle of training (Figure 4, right panel), the groups had reached identical levels of performance. A $2 \times 3$ (groups $X$ trials) ANOVA revealed only a main effect of trials $[F(2,24)=21.24, p<.05]$.

\section{Discussion}

This experiment showed that in addition to the savings shown in the retention task of the first experiment, rats that had undergone hippocampal lesions prior to training could acquire a negative patterning task. In previous studies, we have shown that hippocampal lesions produced in exactly the same manner as in the present series produced 


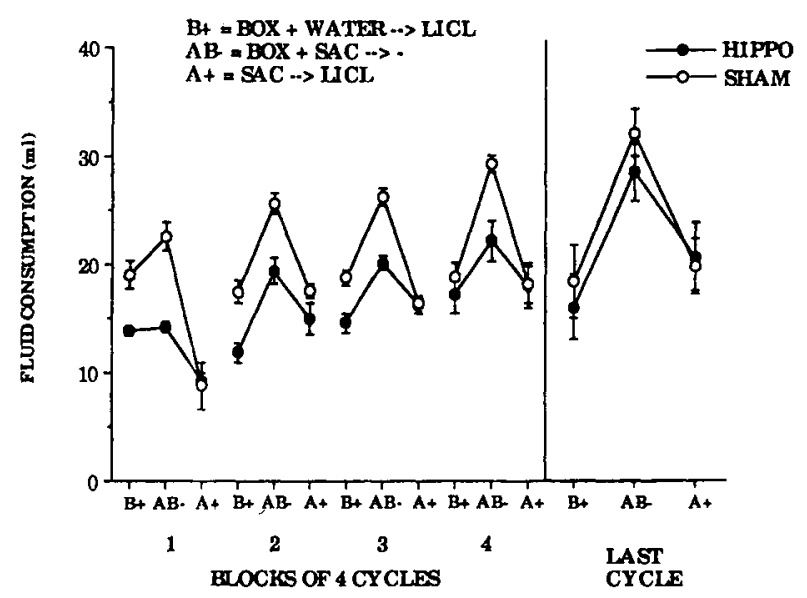

Figure 4. Acquisition of negative patterning in hippocampal and sham lesioned rats. Left panel divides acquisition into four blocks of four cycles. Right panel shows performance on the last cycle $($ Cycle 17). $\mathrm{AB}-=\mathrm{Box}+\mathrm{Sac} \rightarrow-$; $\mathrm{A}+=\mathrm{Sac} \rightarrow \mathrm{LiCl}$; $\mathrm{B}+=\mathrm{Box}+$ Water $\rightarrow \mathrm{LiCl}(n=7$ lesioned and $n=7$ sham rats $)$.

a dramatic impairment on the water maze task (Morris et al., 1982; Skinner, Martin, Harley, et al., 1994), as well as decreased habituation of activity/exploration in an open field test (unpublished data). The negative patterning procedure used in the present experiment involved, first, training the rats on the conditional component, not because these lesions impair conditional learning, but rather because these lesions have effects on contextual learning (Good \& Honey, 1991; Honey \& Good, 1993; Kim \& Fanselow, 1992). Indeed, we have previously shown that the same lesions slowed acquisition on a contextual learning task (Skinner, Martin, Harley, et al.) very similar to the one used in the present study. Although it is possible that hippocampal lesioned rats would have shown a much larger impairment had we trained them on the negative patterning task directly after the lesions, the interpretation of this finding might have been confounded by the fact that they showed deficits in using contextual cues. Thus, in the present study, negative patterning training was carried out once we knew that hippocampal lesioned rats could use contextual cues to guide their behavior. Surprisingly, in the present design, hippocampal lesioned rats were not slower to acquire the conditional task than sham lesioned controls. The one major difference between the conditional task in the present study and that of the previous study (Skinner, Martin, Harley, et al.) was that in the past the animals had to differentiate between two novel contexts that were both different from the home cage. In the present experiment, the rats were exposed only to one novel context, and this might have been an easier problem for the rats to solve. With the addition of $\mathrm{B}+$ trials (i.e., negative patterning training), hippocampal lesioned animals were more impaired than sham controls and showed somewhat more fluid suppression on all trials. By the last cycle of training, however, the two groups were identical. Thus hippocampal lesioned rats are capable of using some contextual cue(s) to solve a negative patterning task.

\section{GENERAL DISCUSSION}

In the present experiments, we developed a negative patterning task using the flavor aversion framework previously employed in conditional discrimination or occasion setting learning (Skinner, Martin, Harley, et al., 1994; Skinner, Martin, Pridgar, \& van der Kooy, 1994). Unmanipulated rats could rapidly acquire the negative patterning task. Although hippocampal lesions did disrupt retention, there was clear evidence of some savings. The same paradigm was used in Experiment 2 to assess acquisition in a larger number of hippocampal lesioned rats. Hippocampal lesioned rats showed good acquisition, suggesting that the hippocampus is not critical for learning a negative patterning task.

A somewhat puzzling finding from the present study is that hippocampal lesions had a greater effect on retention than on acquisition of negative patterning. Whereas hippocampal lesioned rats retained the discrimination between $\mathrm{AB}-$ and $\mathrm{B}+$ trials, drinking significantly more saccharin than tap water in the novel training context, they lost (at least initially) the discrimination between $\mathrm{AB}-$ and $\mathrm{A}+$ trials, consuming equal amounts of saccharin in the novel training context and in the home cage. Perhaps the lesioned rats could not dissociate the two different contexts (home cage vs. novel context). The lesioned rats may not remember the contextual cues, given that contextual information was stored prior to surgery. It is thought that the hippocampus is important for processing contextual information (Good \& Honey, 1991; Honey \& Good, 1993; Kim \& Fanselow, 1992; Young et al., 1994), but it has been shown that preexposure to a context 28 days prior to surgery alleviates any impairment in contextual learning (Kim \& Fanselow, 1992; Young et al., 1994). Behavioral training was initiated more than a month prior to surgery, so this should have been enough time for the animals to consolidate contextual information required for the task. Alternatively, perhaps negative patterning can be acquired by some other brain structure; but if the hippocampus is present, it will dominate, and so we see retention deficits when it is lesioned after training. The retention deficits seen after hippocampal lesions might also have been due to a stimulus generalization deficit. As pointed out by an anonymous reviewer, even if animals retained perfect knowledge of the task, the difference in the world as seen through the eyes of a rat without a hippocampus could have yielded a stimulus generalization decrement. A final possibility is that there may have been interference between the two saccharin trials; to drink or not to drink the same flavored solution may have produced more interference than drinking saccharin and not drinking tap water. The latter interference effect in hippocampal lesioned rats was not seen in the acquisition in Experiment 2, perhaps because the conditional discrimination component of the task (to drink or 
not to drink saccharin) was trained first. Since all of the animals could acquire the conditional component of the task in Experiment 2, we know that the hippocampus is not critical for acquisition of this component.

The present findings do not support the configural association theory as it was originally stated by Sutherland and Rudy (1989), since hippocampal lesioned rats could acquire the negative patterning task. Alvarado and Rudy (1995) have provided a recent explanation of why some configural tasks might not be hippocampal dependent. They show that slight differences in experimental procedures can produce very different behavioral results in a negative patterning task. They compared a standard negative patterning task, in which all trial types were given within a single session, with a split design, in which $\mathrm{A}+$ trials were never given in the same session as were the $\mathrm{B}+$ and $\mathrm{AB}-$ trials. Upon acquisition of the task, further $\mathrm{A}+$ trials were given to both groups. The $\mathrm{A}+$ trials disrupted responding to the $A B$ compound in rats trained on the split design but not in rats trained on the standard design. The generalization from $\mathrm{A}+$ to $\mathrm{AB}$ - would have been higher in the standard version, but training on such a task would eventually decrease this generalization, at least in intact animals. The authors speculated that the hippocampus may play a role when the degree of generalized excitation or interference from the single elements to the compound cue is high. They argued that the degree of generalized excitation would be high in a standard negative patterning task, but not in the split design. One prediction from their explanation of the data is that a negative patterning task in which animals are given only one trial per day (or a split design) might be soluble by hippocampal lesioned animals. Giving one trial per day might decrease interference or generalized excitation to a sufficient degree so that hippocampal involvement might not be essential for acquisition of a negative patterning task. Indeed, in Experiment 2, hippocampal lesioned rats could acquire a negative patterning task with a $24-\mathrm{h}$ intertrial interval.

In a recent "reconfiguration" of the original theory, Rudy and Sutherland (1995) have considered these issues. Their new claim is that the hippocampus contributes to configural learning by enhancing the activation or salience of configural representations constructed in other areas of the cortex, essentially reducing generalization between the compound and its component elements. One prediction from the revised theory is that the impairment typically seen on negative patterning tasks in hippocampal lesioned animals can be reduced or eliminated by varying the parameters of the task to reduce the generalization between the components of the problem. This was accomplished in the present design by introducing a 24-h intertrial interval. Thus, the present data are consistent with the revised theory (Rudy \& Sutherland, 1995), although it is worth stating that this new theory is now more of a perceptual theory than a learning theory.

The data from the present experiments do not fit the declarative (relational)/procedural dissociation of memory systems (Eichenbaum et al., 1992, 1994). The dis- tinction between this theory and the old configural theory is somewhat ambiguous, given that both accounts deal with associations among multiple elements. Relational theory, like the old configural theory, has trouble explaining why some apparently relational or configural tasks are hippocampal dependent and others are hippocampal independent. It is not clear that relational theories would predict differences between a within-session negative patterning task and a split design (as in the present study, in which rats were given one trial per day). Such slight procedural variations should not change the relationship of one stimulus to others in the same task.

The present data are in line with several recent reports showing that hippocampal lesioned animals can learn complex tasks (Davidson et al., 1993; Gallagher \& Holland, 1992; Whishaw \& Tomie, 1991). Together, these data pose a challenge to existing relational and configural theories of learning and memory.

\section{REFERENCES}

Aggleton, J. P., Kentridge, R. W., \& Sembi, S. (1991). Lesions of the fornix but not the amygdala impair acquisition of concurrent discriminations by rats. Behavioural Brain Research, 48, 103-112.

Alvarado, M. C., \& Rudy, J. W. (1992). Some properties of configural learning: An investigation of the transverse-patterning problem. Journal of Experimental Psychology: Animal Behavior Processes, 18, 145-153.

Alvarado, M. C., \& Rudy, J. W. (1995). A comparison of "configural" discrimination problems: Implications for understanding the role of the hippocampal formation in learning and memory. Psychobiology, 23, 178-184.

Cohen, N. J., \& SQuire, L. R. (1980). Preserved learning and retention of pattern-analyzing skill in amnesia: Dissociation of knowing how and knowing that. Science, 210, 207-210.

Davidson, T. L., McKernan, M. G., \& Jarrard, L. E. (1993). Hippocampal lesions do not impair negative patterning; A challenge to configural association theory. Behavioral Neuroscience, 107, 227 234.

Eichenbaum, H., Fagan, A., Mathews, P., \& Cohen, N. J. (1988). Hippocampal system dysfunction and odor discrimination learning in rats: Impairment or facilitation depending on representational demands. Behavioral Neuroscience, 102, 331-339.

Eichenbaum, H., Otto, T., \& Cohen, N. J. (1992). The hippocampuswhat does it do? Behavioral \& Neural Biology, 57, 2-36.

Eichenbaum, H., Otto, T., \& Cohen, N. J. (1994). Two functional components of the hippocampal memory system. Behavioral \& Brain Sciences, 17, 449-518.

Eichenbaum, H., Stewart, C., \& Morris, R. G. M. (1990). Hippocampal representation in place learning. Journal of Neuroscience, 10 , 3531-3542.

Gallagher, M., \& Holland, P. C. (1992). Preserved configural learning and spatial learning impairment in rats with hippocampal damage. Hippocampus, 2, 81-88.

GoOD, M., \& HONEY, R. C. (1991). Conditioning and contextual retrieval in hippocampal rats. Behavioral Neuroscience, 105, 499 509.

HIRSH, R. (1974). The hippocampus and contextual retrieval of information from memory: A theory. Behavioral Biology, 12, 421-444.

HONEY, R. C., \& Good, M. (1993). Selective hippocampal lesions abolish the context specificity of latent inhibition and conditioning. $B e$ havioral Neuroscience, 107, 23-33.

Kim, J. J., \& FANSELOW, M. S. (1992). Modality-specific retrograde amnesia of fear. Science, 256, 675-677.

McDonald, R. J., \& White, N. M. (1993). A triple dissociation of memory systems: Hippocampus, amygdala, and dorsal striatum. Behavioral Neuroscience, 107, 3-22.

Morris, R. G. M., Garrud, P., Rawlins, J. N. P., \& O'Keefe, J. (1982). 
Place navigation impaired in rats with hippocampal lesions. Nature, 297, 681-683.

Morris, R. G. M., Schenk, F., Tweedie, F., \& Jarrard, L. E. (1990). Ibotenate lesions of the hippocampus and/or subiculum: Dissociating components of allocentric spatial learning. European Journal of Neuroscience, 2, 1016-1028.

O'Keefe, J., \& Nadel, L. (1979). Precis of O'Keefe \& Nadel's The hippocampus as a cognitive map. Behavioral \& Brain Sciences, 2, 487-533.

Olton, D. S., Becker, J. T., \& Handelmann, G. E. (1980). Hippocampal function: Working memory or cognitive mapping? Physiological Psychology, 8, 239-246.

RafFaele, K. C., \& Olton, D. S. (1988). Hippocampal and amygdaloid involvement in working memory for nonspatial stimuli. Behavioral Neuroscience, 102, 349-355.

Ridley, R. M., Timothy, C. J., MacLean, C. J., \& BaKer, H. F. (1995), Conditional learning and memory impairments following neurotoxic lesion of the CAl field of the hippocampus. Neuroscience, 67, 263-275.

Rudy, J. W., \& SuTherland, R. J. (1989). The hippocampal formation is necessary for rats to learn and remember configural discriminations. Behavioural Brain Research, 34, 97-109.

RuDY, J. W., \& SUTHERLAND, R. J. (1995). Configural association theory and the hippocampal formation: An appraisal and reconfiguration. Hippocampus, 5, 375-389.

Selden, N. R. W., Everitt, B. J., Jarrard, L. E., \& Robins, T. W. (1991). Complementary roles for the amygdala and hippocampus in aversive conditioning to explicit and contextual cues. Neuroscience, 42, 335-350.

Skinner, D. M., Martin, G. M., Harley, C., Kolb, B., Pridgar, A., BECHARA, A., \& VAN DER KOOY, D. (1994). Acquisition of conditional discriminations in hippocampal lesioned and decorticated rats: Evidence for learning that is separate from both simple classical conditioning and configural learning. Behavioral Neuroscience, $108,911-926$

Skinner, D. M., Martin, G. M., Pridgar, A., \& van der Kooy, D. (1994). Conditional control of fluid consumption in an occasion setting paradigm is independent of Pavlovian associations. Learning \& Motivation, 25, 368-400.

SQUIRE, L. R. (1986). Mechanisms of memory. Science, 232, 1612 1619.

Sutherland, R. J., \& MCDonald, R. J. (1990). Hippocampus, amyg- dala, and memory deficits in rats. Behavioural Brain Research, 37, 57-79.

Sutherland, R. J., \& Rudy, J. W. (1988). Place learning in the Morris place navigation task is impaired by damage to the hippocampal formation even if the temporal demands are reduced. Psychobiology, 16, 157-163.

Sutherland, R. J., \& RudY, J. W. (1989). Configural association theory: The role of the hippocampal formation in learning, memory, and amnesia. Psychobiology, 17, 129-144.

Sutherland, R. J., WhishaW, I. Q., \& KolB, B. (1983). A behavioral analysis of spatial localization following electrolytic, kainate- or colchicine-induced damage to the hippocampal formation in the rat. Behavioural Brain Research, 7, 133-153.

Wan, R. Q., PANG, K., \& Olton, D. S. (1994). Hippocampal and amygdaloid involvement in nonspatial and spatial working memory in rats: Effects of delay and interference. Behavioral Neuroscience, 108, 866-882.

WhISHAW, I. Q., \& Tomie, J. (1991). Acquisition and retention by hippocampal rats of simple, conditional, and configural tasks using tactile and olfactory cues: Implications for hippocampal function. Behavioral Neuroscience, 105, 787-797.

Whishaw, I. Q., \& TomIE, J. (1995). Rats with fimbria-fornix lesions can acquire and retain a visual-tactile transswitching (configural) task. Behavioral Neuroscience, 109, 607-612.

Whishaw, I. Q., Tomie, J., \& KolB, B. (1992). Ventrolateral prefrontal cortex lesions in rats impair the acquisition and retention of a tactile-olfactory configural task. Behavioral Neuroscience, 106, $597-603$.

WOODBURY, C. B. (1943). The learning of stimulus patterns by dogs. Journal of Comparative Psychology, 35, 29-40.

YEE, B. K., \& RaWlins, J. N. P. (1994). The effects of hippocampal formation ablation or fimbria-fornix section on performance of a nonspatial radial arm maze task by rats. Journal of Neuroscience, 14, 3766-3774.

YounG, S. L., Bohenek, D. L., \& Fanselow, M. S. (1994). NMDA processes mediate anterograde amnesia of contextual fear conditioning induced by hippocampal damage: Immunization against amnesia by context preexposure. Behavioral Neuroscience, 108, 19-29.

(Manuscript received July 1, 1996; revision accepted for publication November $29,1996$. 